\title{
Metabolic Dysfunction-Associated Fatty Liver Disease Increases the Risk of Gastroesophageal Reflux Symptoms
}

\author{
Yuan $\mathrm{He}^{\mathrm{l}, 2}$ \\ Zhi-Jun Duan' \\ Cheng-Fang Wang ${ }^{2}$ \\ Yu-Shan Wei ${ }^{3}$ \\ Ming-Xu Cai ${ }^{2}$ \\ 'Department of Gastroenterology, The \\ First Affiliated Hospital of Dalian Medical \\ University, Dalian, II60I I, People's \\ Republic of China; ${ }^{2}$ Healthcare \\ Management Center, The First Affiliated \\ Hospital of Dalian Medical University, \\ Dalian, II60II, People's Republic of \\ China; ${ }^{3}$ Department of the Scientific \\ Research Management, The First \\ Affiliated Hospital of Dalian Medical \\ University, Dalian, II60I I, People's \\ Republic of China
}

\begin{abstract}
Objective: The present study aimed to explore the relationship between metabolic dysfunction-associated fatty liver disease (MAFLD) and gastroesophageal reflux symptoms (GERS). Methods: The present study was a cross-sectional observational study. The study population was 3002 subjects from a single hospital who underwent a health checkup from September 1, 2019, to December 31, 2020. The diagnosis of MAFLD was based on the diagnosis of fatty liver in the subject by ultrasound or computed tomography (CT) and the presence of one of the following conditions: overweight or obesity (body mass index $[\mathrm{BMI}] \geq 23$ ), type 2 diabetes mellitus, and metabolic abnormalities. The subjects were divided into the GERS group $(n=305)$ and the non-GERS group $(n=2697)$ based on the presence or absence of GERS, based on the GerdQ score.

Results: The prevalence of MAFLD was significantly higher in the GERS group than in the non-GERS group $(p=0.001)$. In the univariate analysis of risk factors for GERS, MAFLD was identified as a risk factor for GERS (OR 1.5; 95\% CI 1.176-1.913; $p=0.001)$. With adjustment of confounding factors such as BMI, waist circumference, lipid levels, and blood pressure, the correlation between MAFLD and GERS was attenuated but still significant (OR $1.408 ; 95 \%$ CI $1.085-1.826 ; \mathrm{p}=0.010$ ).
\end{abstract}

Conclusion: MAFLD might be an independent risk factor for GERS.

Keywords: gastroesophageal reflux symptoms, GERD, metabolic dysfunction-associated fatty liver disease, body mass index, obesity, questionnaire

\section{Introduction}

Non-alcoholic fatty liver disease (NAFLD) is by far the most common liver disease globally, and it is reported to affect $20-30 \%$ of the population worldwide. ${ }^{1}$ Studies have shown that NAFLD can not only progress to cirrhosis and even liver cancer but is also strongly correlated with an increased risk of serious extrahepatic diseases such as cardiovascular disease (CVD) and metabolic syndrome (MetS) and that patients with NAFLD are twice as likely to die from CVD than from liver disease. $^{1,2}$ Therefore, a new concept, named metabolic dysfunction-associated fatty liver disease (MAFLD), has been proposed recently. ${ }^{3}$ The diagnosis of MAFLD no longer requires the exclusion of other hepatic etiologies such as excessive alcohol consumption, hepatitis virus infection, and autoimmune liver disease. Metabolic dysfunction is required for the diagnosis of MAFLD. ${ }^{4}$

Gastroesophageal reflux disease (GERD) is a common gastrointestinal disorder characterized clinically by reflux of gastric contents into the esophagus,
Correspondence: Zhi-Jun Duan Department of Gastroenterology, The First Affiliated Hospital of Dalian Medical University, Dalian, II60I I, People's Republic of China

$\mathrm{Tel} / \mathrm{Fax}+864$ II 83635963

Email duanzhijun12@outlook.com; cathydoctor@sina.com 
causing symptoms such as heartburn, reflux, and dysphagia. ${ }^{5}$ The global prevalence of GERD has been reported to be approximately $13 \%,{ }^{6,7}$ and the poor therapeutic outcome has negatively impacted the quality of life in patients. It is found from previous studies ${ }^{8-11}$ that MetS is both an independent risk factor for GERD and a well-known risk factor for fatty liver disease (FLD). ${ }^{12}$ Hence, we wondered whether there existed an association between GERD and FLD. However, there are not many studies on the relationship between GERD and FLD,${ }^{13-21}$ and the findings on the relationship are inconsistent. Therefore, a cross-sectional observational study was conducted to investigate the relationship between gastroesophageal reflux symptoms (GERS) and MAFLD diagnosed by the new criteria to provide new ideas for the prevention and treatment of GERD.

\section{Materials and Methods}

A cross-sectional observational study was conducted in compliance with ethical guidelines and with due regard to patient privacy. Informed consent for the application of the questionnaire and testing data for the study was obtained from all participants. The protocol was approved by the Ethical Committee of the First Affiliated Hospital of Dalian Medical University, Dalian, China.

\section{Questionnaire Survey}

First, all subjects who volunteered to participate in the study completed three questionnaires under the guidance of a trained physician.

\section{Lifestyle Questionnaire}

The contents included the age, gender, educational background (undergraduate, above undergraduate, or below undergraduate), smoking (yes or no, and when the participant indicated "yes," this meant the participant smoked $\geq 1$ cigarette per day for six consecutive or cumulative months), alcohol drinking (yes or no, and for "yes," this meant daily alcohol consumption $>25 \mathrm{~g}$ for males or $>15$ $\mathrm{g}$ for females for six consecutive or cumulative months), medical history (previous and current illness and related treatment), physical activity (little exercise, $<150$ minutes/ week, or $\geq 150$ minutes/week), daily sleep duration $(<5$ hours, 5-7 hours, $>7$ hours), the habit of overeating (yes or no), irregularity in eating three meals (yes or no), the habit of lying flat on one's back after eating (within 30 minutes) (yes or no) in the subject.

\section{GerdQ Questionnaire}

All participants were asked to fill out the GerdQ questionnaire on their own. GerdQ is a simple questionnaire developed as a part of the Diamond study. ${ }^{22,23}$ It asks the participants to score the number of days with symptoms (heartburn, regurgitation, epigastric pain, nausea, sleep disturbances, or the use of over-The-counter medications due to symptoms of reflux, epigastric pain, and nausea) during the previous seven days. It uses a four-graded Likert scale (0-3) to score the frequency of four positive predictors of GERD (heartburn, regurgitation, sleep disturbance due to reflux symptoms, or use of over-Thecounter medications for reflux symptoms) and a reversed Likert scale (3-0) for two negative predictors of GERD (epigastric pain and nausea). The results of the questionnaire emerge as a score between 0 and 18. GerdQ has been used in several studies. ${ }^{24-27}$ Most validation studies of GerdQ have presented the cut-off as 8. A high likelihood of GERD is indicated if the score is 8 or higher.

\section{Hospital Anxiety and Depression Scale (HADS)}

We used the Hospital Anxiety and Depression Scale $(\mathrm{HADS})^{28}$ to measure the participants' mental state. This scale includes 14 items (seven items for anxiety and seven items for depression). The response descriptors of all items are: definitely (score 3); sometimes (score 2); not much (score 1); not at all (score 0); while the items 7 and 10 are scored reversely. A score of 11 or greater indicates anxiety or depression, 8-10 indicates a borderline case, and 7 or lower indicates no signs of anxiety or depression. The HADS has been supported in some studies. ${ }^{29-32}$

\section{Anthropometrics and Laboratory Measurement Methods}

\section{Anthropometrics}

(1)Method for blood pressure measurement: the Omron desktop medical electronic blood pressure monitor (Omron HBP-9020 type) was adopted to measure blood pressure for the subject. The subject was measured three times, and the average value was taken. (2) Method for body mass index (BMI) measurement: subjects were dressed in light clothing, and the BMI results were automatically output by the Hyporda ultrasonic automatic height and weight meter (TCS-135C-RTB). (3) Waist circumference measurement method: the waist circumference was measured at the midpoint of the line between the lower edge of the rib cage and the iliac ridge. ${ }^{33} \mathrm{An}$ assigned nurse performed all the above measurements. 


\section{Laboratory Indicators}

Venous blood samples were collected early in the morning after the subject had fasted for at least ten hours. The laboratory indicators included the metabolic indicators adopted in the diagnosis of MAFLD: total cholesterol (TC), triglycerides (TG), low-density lipoprotein cholesterol (LDL-C), high-density lipoprotein cholesterol (HDLC), serum uric acid (UA), fasting plasma glucose (FPG), glycated hemoglobin (HbAlc), insulin (IRI), and C-reactive protein (CRP). It also included indicators reflecting the hepatic function: aspartate aminotransferase (AST), alanine aminotransferase (ALT), glutamate aminotransferase $(\gamma-\mathrm{GT})$, alkaline phosphatase (ALP), total bilirubin (T-Bil), direct bilirubin (D-Bil), bile acids, and albumin (ALB). These parameters were measured with the adoption of the clinical laboratory assay standards.

\section{Helicobacter pylori $(\mathrm{Hp})$ Detection}

All subjects took ${ }^{14} \mathrm{C}$ urea capsules orally on an empty stomach, and the subjects were instructed by a designated nurse to blow on an expiratory card for 2-5 minutes after sitting for 15 minutes. The yellow color of the indicator window on the card indicated the completion of the collection. Then the expiratory card was inserted into the Hp testing instrument (Hedwig HUBT-20A), and a value of $\geq 100 \mathrm{dpm} / \mathrm{mmol}$ indicated positive for Hp.

\section{The Diagnostic Methods for MAFLD}

The abdominal ultrasound or abdominal computed tomography (CT) scan was conducted in all subjects. The diagnosis of MAFLD was based on imaging diagnosis of hepatic steatosis together with one of the following conditions: overweight/obesity, diabetes mellitus, and metabolic dysfunction., ${ }^{3,4}$ Overweight was defined as a BMI of $\geq 23 \mathrm{~kg} / \mathrm{m}^{2}$ in Asians. Metabolic abnormalities were diagnosed by the presence of two or more of the following conditions: (1) waist circumference $\geq 90 / 80 \mathrm{~cm}$ in Asian males/females; (2) serum $\mathrm{TG} \geq 1.7 \mathrm{mmol} / \mathrm{L}$ or with the administration of lipid-lowering drugs; (3) blood pressure $\geq 130 / 85 \mathrm{mmHg}$ with the administration of blood pressurelowering drugs; (4) plasma HDL-C $<1.0$ in males or 1.3 $\mathrm{mmol} / \mathrm{L}$ in females or with the administration of lipidlowering drugs; (5) pre-diabetes FPG within the range of 5.6 6.9 $\mathrm{mmol} / \mathrm{L}$ or postprandial two-hour blood glucose within the range of $7.8 \sim 11.0 \mathrm{mmol} / \mathrm{L}$ or $\mathrm{HbAlc}$ within the range of $7 \% \sim 6.4 \%$; (6) IRI by the homeostasis model $\geq 2.5$; (7) serum ultra-sensitive CRP $>2 \mathrm{mg} / \mathrm{L}$. In the present study, 952 subjects were diagnosed with MAFLD.

\section{Study Subjects and Experiment Design}

A total of 3605 subjects between the ages of 18 and 80 voluntarily participated in the present study. These subjects all participated in the physical examination at the Health Examination Center of the First Affiliated Hospital of Dalian Medical University, China, from September 1, 2019, to December 31, 2020. Pregnant women were not included in this study. We excluded 571 subjects, including incomplete data $(\mathrm{n}=406)$, a history of malignancy $(\mathrm{n}=53)$, a history of gastric or esophageal surgery $(n=12)$, a history of cholecystitis and pancreatitis ( $\mathrm{n}=91$ ), and administration of drugs such as tamoxifen, amoxicillin, valproate, methotrexate, and glucocorticoids that might affect the lipid metabolism or cause gastrointestinal symptoms $(n=9)$. The final number of eligible subjects was 3034 . Of these, 32 had hepatic steatosis confirmed by abdominal ultrasound or CT scan, but without obesity, metabolic dysfunction, or diabetes mellitus, and they were not included in the present study. Of the final 3002 participants, those with a GerdQ score $\geq 8$ were defined as the GERS group $(n=305)$, and those with a GerdQ score of $<8$ were defined as the non-GERS group $(n=2697)$ based on the GerdQ scale.

\section{Statistical Methods}

The SPSS 26.0 software was adopted. The measurement data that satisfied the normal distribution were expressed as " $\overline{\mathrm{x}} \pm \mathrm{s}$," and the dependent sample $t$-test was adopted for comparison between groups. The measurement data that did not satisfy the normal distribution were expressed by the median (P25, P75), and the Mann-Whitney test was used to compare the differences between the two groups. The countable data were expressed as $\mathrm{n}(\%)$, and the Chisquare test and Fisher's test were used to compare the differences between the two groups. Univariate and multivariate analyses of risk factors for GERS were conducted with the adoption of the binary logistic regression analysis to assess the relationship between MAFLD and GERS before and after adjustment for the confounding factors. $\mathrm{P}<0.05$ was considered statistically significant.

\section{Results}

The background characteristics of the participants are demonstrated in Table 1. Among the 3002 subjects, 305 were positive for GERS with a prevalence of $10.16 \%$. There was no significant difference in the gender ratio in the GERS group compared to the non-GERS group, while 
Table I The Background Characteristics of the Study Population $(n=3002)$

\begin{tabular}{|c|c|c|c|c|}
\hline Characteristic Variable & GERS Group $(n=305)$ & Non-GERS Group $(n=2697)$ & Chi-Square /t-value & p-value \\
\hline Male in gender & 149 (48.9\%) & 1324 (49.1\%) & 0.006 & 0.952 \\
\hline Age & $41.5 \pm 9.8$ & $39.1 \pm 9.5$ & 4.464 & $<0.001$ \\
\hline Educational background & & & 5.075 & 0.079 \\
\hline Undergraduate & $185(60.7 \%)$ & 1670 (61.9\%) & & \\
\hline Below undergraduate & $68(22.3 \%)$ & 474 (17.6\%) & & \\
\hline Above undergraduate & $52(17.0 \%)$ & $553(20.5 \%)$ & & \\
\hline Smoking & 58 (19.0\%) & $464(17.2 \%)$ & 0.626 & 0.426 \\
\hline Alcohol drinking & $5(1.6 \%)$ & $42(1.6 \%)$ & $0.018^{*}$ & 0.809 \\
\hline Exercise & & & 1.266 & 0.531 \\
\hline Hardly any exercise & $100(32.8 \%)$ & $801(29.7 \%)$ & & \\
\hline$<150 \mathrm{~min} /$ week & $119(39.0 \%)$ & $1090(40.4 \%)$ & & \\
\hline$>150 \mathrm{~min} /$ week & $86(28.2 \%)$ & $806(29.9 \%)$ & & \\
\hline Sleeping duration & & & 8.849 & 0.012 \\
\hline$<5$ hours & II (3.6\%) & $58(2.2 \%)$ & & \\
\hline 5-7 hours & $186(61.0 \%)$ & 1467 (54.4\%) & & \\
\hline$>7$ hours & $108(35.4 \%)$ & 1172 (43.5\%) & & \\
\hline Habit of overeating & $74(24.3 \%)$ & 491 (18.2\%) & 6.579 & 0.013 \\
\hline Irregular eating & $63(20.7 \%)$ & $437(16.2)$ & 3.913 & 0.052 \\
\hline The habit of lying flat after a meal & $164(53.8 \%)$ & 1157 (42.9\%) & 13.142 & $<0.001$ \\
\hline GerdQ Score & $8.9 \pm 1.3$ & $5.9 \pm 0.7$ & 62.378 & $<0.001$ \\
\hline Hypertension & $32(10.5 \%)$ & 145 (5.4\%) & 12.923 & 0.001 \\
\hline Diabetes mellitus & $6(2.0 \%)$ & $48(1.8 \%)$ & 0.055 & 0.819 \\
\hline Helicobacter pylori infection & $86(28.2 \%)$ & $744(27.6 \%)$ & 0.051 & 0.839 \\
\hline MAFLD & $122(40.0 \%)$ & $830(30.8 \%)$ & 10.768 & 0.001 \\
\hline Anxiety score & $6.1 \pm 3.6$ & $4.5 \pm 3.2$ & 8.115 & $<0.001$ \\
\hline Depression score & $5.3 \pm 3.9$ & $4.3 \pm 3.2$ & 5.347 & $<0.001$ \\
\hline BMI & $25.2 \pm 3.8$ & $24.7 \pm 3.8$ & 2.332 & 0.020 \\
\hline Waist Circumference & $83.4 \pm 10.9$ & $81.5 \pm 11.2$ & 2.908 & 0.004 \\
\hline SBP & $124.4 \pm 15.7$ & $122.2 \pm 15.4$ & 2.389 & 0.017 \\
\hline DBP & $77.1 \pm 12.4$ & $75.3 \pm 11.5$ & 2.470 & 0.014 \\
\hline Serum uric acid & $338.8 \pm 87.4$ & $338.4 \pm 96.1$ & 0.064 & 0.949 \\
\hline Blood glucose & $5.105 \pm 1.095$ & $5.054 \pm 0.957$ & 0.873 & 0.383 \\
\hline LDL-C & $2.734 \pm 0.643$ & $2.698 \pm 0.668$ & 0.887 & 0.375 \\
\hline HDL-C & $1.249 \pm 0.321$ & $1.279 \pm 0.297$ & -1.626 & 0.104 \\
\hline TG & $\mathrm{I} .22(0.8 \mathrm{I}, \mathrm{I} .8 \mathrm{I})$ & $1.06(0.75,1.61)$ & & 0.002 \\
\hline TC & $5.042 \pm 0.904$ & $4.995 \pm 0.933$ & 0.835 & 0.404 \\
\hline ALT & $18(13,29)$ & $18(12,28)$ & & 0.302 \\
\hline AST & $21.0 \pm 8.2$ & $20.7 \pm 9.1$ & 0.634 & 0.526 \\
\hline Albumin & $45.7 \pm 2.4$ & $46.2 \pm 2.5$ & -3.153 & 0.002 \\
\hline ALP & $70.0 \pm 18.0$ & $68.2 \pm 17.7$ & 1.724 & 0.085 \\
\hline$\gamma$-GT & $22(15,34)$ & $21(14,34)$ & & 0.192 \\
\hline TBIL & $13.6 \pm 5.1$ & $13.8 \pm 5.2$ & -0.781 & 0.435 \\
\hline DBIL & $3.8 \pm 1.6$ & $3.9 \pm 1.6$ & -1.681 & 0.093 \\
\hline BA & $2.2(1.4,3.2)$ & $2.2(1.5,0.2)$ & & 0.513 \\
\hline
\end{tabular}

Notes: Data are shown as the mean \pm SD, the median (P25, P75) or number (\%). " Fisher's test.

Abbreviations: MAFLD, Metabolic dysfunction-associated fatty liver disease; BMI, body mass index; SBP, systolic blood pressure; DBP, diastolic blood pressure; LDL-C, low density lipoprotein-cholesterol; HDL-C, high density lipoprotein-cholesterol; TG, triglycerides; TC, total cholesterol; ALT, alanine aminotransferase; AST, aspartate aminotransferase; ALP, alkaline phosphatase; $\gamma$-GT, gamma-glutamyltranspetidase; TBIL, total bilirubin; DBIL, direct bilirubin; BA, bile acids.

the age was higher in the GERS group $(41.5 \pm 9.8$ years $)$ than in the non-GERS group $(39.1 \pm 9.5, \mathrm{p}<0.001)$. Regarding lifestyle habits, the GERS group had a shorter sleep duration and a higher proportion of subjects who had the habit of lying flat on their backs and overeating. In contrast, there were no significant differences between the 
two groups in terms of educational background, smoking, alcohol consumption, and physical activity. Concerning the psychological status, anxiety and depression scores were significantly higher in the GERS group than in the non-GERS group $(\mathrm{p}<0.001)$. There existed a significant difference in the prevalence of MAFLD between the two groups $(p=0.001)$. The prevalence of hypertension was significantly higher in the GERS group than in the nonGERS group. BMI and waist circumference, which represent general and central obesity, respectively, were also higher in the GERS group than in the non-GERS group. In addition, there existed statistically significant differences in TG and ALB levels between the two groups. In contrast, there were no significant differences in the history of diabetes mellitus, Hp infection, or other laboratory indicators between the two groups.

Univariate regression analysis was adopted to assess the relationship between GERS and MAFLD (as shown in Table 2). It suggested that MAFLD was a risk factor for GERS (OR 1.5; 95\% CI 1.176-1.913; p = 0.001). In addition, according to the present study, the risk factors for GERS included the following indicators: age, short sleep duration, the habit of overeating, a habit of lying flat after meals, anxiety, depression, hypertension, BMI, waist circumference, TG, and ALB.

Then, multivariate binary logistic regression analysis (conditional backward method, as shown in Table 3) was adopted to screen out the independent risk factors for
GERS. A 9-step stepwise adjustment for confounding factors finally yielded MAFLD as an independent risk factor for GERS (OR 1.408; 95\% CI 1.085-1.826; $\mathrm{p}=$ 0.010). Meanwhile, it was found that after adjusting for the confounding factors, BMI and waist circumference, representing obesity, were not identified as risk factors for GERS, while age (OR 1.027; 95\% CI 1.012-1.041; $\mathrm{p}<0.001$ ), history of hypertension (OR 1.572; 95\% CI 1.010-2.448; $\mathrm{p}=0.045$ ), anxiety (OR 1.147; 95\% CI 1.108-1.187; p < 0.001), lifestyle habits of lying flat after meals (OR 1.481; 95\% CI 1.154-1.902; $\mathrm{p}=$ 0.002 ) and ALB levels (OR 0.930; 95\% CI 0.882$0.980 ; p=0.007)$ were independently correlated with GERS.

Finally, the GerdQ score in the population diagnosed with MAFLD was compared with that in the non-MAFLD population (as shown in Figure 1), and it was found that the GerdQ score was significantly higher in the MAFLD population $(6.471 \pm 1.295)$ than in the non-MAFLD population $(6.137 \pm 1.152, \mathrm{t}=4.689, \mathrm{p}<0.001)$.

\section{Discussion}

In the present study, 3002 subjects for health checkups were included, and risk factors for GERS were systematically sorted in terms of laboratory indicators, psychological status, and lifestyle (including smoking, alcohol consumption, physical activity, dietary habits, and sleep habits). It was found that MAFLD increased the risk of

Table 2 Results of Univariate Logistic Regression Analysis of the Risk Factors for GERG Like Symptoms (Enter Method)

\begin{tabular}{|l|l|l|l|}
\hline Independent Variable & B-value & p-value & OR (95\% CI) \\
\hline MAFLD & 0.405 & 0.001 & $1.5(1.176 \sim 1.913)$ \\
Age & 0.027 & $<.001$ & $1.028(1.015 \sim 1.040)$ \\
Sleeping duration & & 0.013 & \\
$\quad>7$ hours & 0.722 & 0.036 & $2.058(1.049 \sim 4.038)$ \\
$\quad<5$ hours & 0.319 & 0.012 & $1.376(1.072 \sim 1.765)$ \\
$5-7$ hours & 0.364 & 0.011 & $1.439(1.088 \sim 1.903)$ \\
Habit of overeating & 0.437 & $<0.001$ & $1.548(1.221 \sim 1.963)$ \\
The habit of lying flat after a meal & 0.724 & $<0.001$ & $2.063(1.379 \sim 3.086)$ \\
History of hypertension & 0.134 & $<0.001$ & $1.143(1.106 \sim 1.182)$ \\
Anxiety score & 0.088 & $<0.001$ & $1.092(1.057 \sim 1.128)$ \\
Depression score & 0.036 & 0.020 & $1.037(1.006 \sim 1.068)$ \\
BMI & 0.015 & 0.004 & $1.015(1.005 \sim 1.026)$ \\
Waist circumference & 0.009 & 0.017 & $1.009(1.002 \sim 1.017)$ \\
Systolic blood pressure & 0.013 & 0.014 & $1.013(1.003 \sim 1.023)$ \\
Diastolic blood pressure & 0.148 & 0.004 & $1.159(1.048 \sim 1.282)$ \\
TG & -0.077 & 0.002 & $0.926(0.881 \sim 0.973)$ \\
Albumin & &
\end{tabular}

Abbreviations: MAFLD, metabolic dysfunction-associated fatty liver disease; BMI, body mass index; TG, triglycerides. 
Table 3 Results of Multivariate Logistic Regression Analysis of the Risk Factors for Gastroesophageal Reflux Symptoms (Conditional Backward Method)

\begin{tabular}{|l|l|l|l|}
\hline Independent Variable & B-value & p-value & OR (95\% CI) \\
\hline MAFLD & 0.342 & 0.010 & $1.408(1.085 \sim 1.826)$ \\
Age & 0.026 & $<0.001$ & $1.027(1.012 \sim 1.04 I)$ \\
The habit of lying flat after a meal & 0.393 & 0.002 & $1.48 I(1.154 \sim 1.902)$ \\
Anxiety score & 0.137 & $<0.001$ & $1.147(1.108 \sim 1.187)$ \\
History of hypertension & 0.452 & 0.045 & $1.572(1.010 \sim 2.448)$ \\
Albumin & -0.073 & 0.007 & $0.930(0.882 \sim 0.980)$ \\
\hline
\end{tabular}

Abbreviation: MAFLD, metabolic dysfunction-associated fatty liver disease.

occurrence of GERS and remained an independent risk factor for GERS after adjusting for multiple confounding factors.

A study by Fujiwara et $\mathrm{al}^{17}$ concluded that the high prevalence of GERS in patients with NAFLD was correlated with BMI. Min et al $^{14}$ stated that NAFLD was not independently correlated with the risk of developing reflux esophagitis but was the result of increased BMI associated with NAFLD. They concluded that obesity, which was often combined in patients with fatty liver, increased the intra-abdominal pressure and decreased the lower esophageal sphincter (LES) pressure, thus increasing the chance of reflux. It was revealed in the present study that BMI, waist circumference, and the prevalence of MAFLD were significantly higher in the GERS group than in the nonGERS group, and all three were significant risk factors for GERD (OR 1.5 for MAFLD, 95\% CI 1.176-1.913, $\mathrm{p}=$ 0.001). After BMI, waist circumference, and other factors were gradually adjusted as confounding factors, the

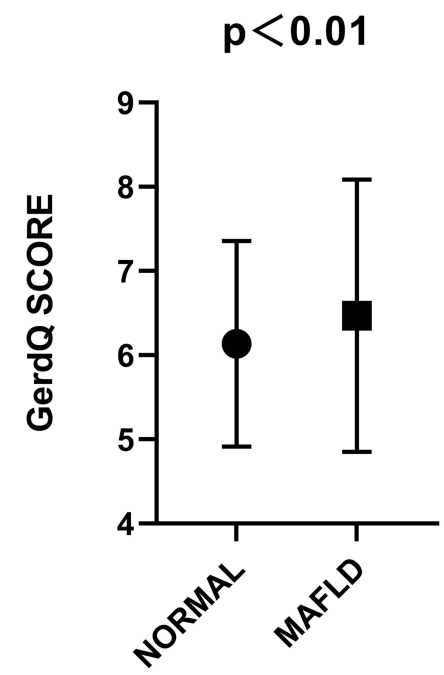

Figure I Comparison of GerdQ scores between the MAFLD group and nonMAFLD group.

Abbreviation: MAFLD, metabolic dysfunction-associated fatty liver disease. correlation between MAFLD and GERS was weakened but still significant (OR 1.408; 95\% CI 1.085-1.826; $\mathrm{p}=$ $0.010)$. This indicated that MAFLD was still independently correlated with GERS, and the effect of MAFLD on GERS might be greater than that of BMI, which represented general obesity, and waist circumference, which represented central obesity.

In addition, the fatty liver might contribute to the development of GERD through several mechanisms: (1) fatty liver might be correlated with GERD through dysfunction of the autonomic nervous system. Some studies have shown that patients with NAFLD have a higher prevalence of autonomic dysfunction. ${ }^{34,35}$ And autonomic dysfunction might lead to abnormal gastric and esophageal motility, thus promoting the development of GERD. (2) Gehrke et $\mathrm{al}^{36}$ introduced the concept of metabolic inflammation, which they suggested might be a key process driving the development of fatty liver and extrahepatic disease. These inflammations might cause systemic effects that could be detected by systemic alterations in immune cell subsets and humoral factors. It has been found that serum levels of the inflammatory factors interleukin-1 (IL1) and interleukin-6 (IL-6) are elevated in patients with fatty liver, ${ }^{37,38}$ while IL-1 and IL- 6 can weaken the contractile function of the LES and thus worsen the reflux. ${ }^{39}$ The above studies demonstrated that the metabolic inflammatory response in the fatty liver might contribute to the development of GERD.

In addition to identifying MAFLD as an independent risk factor for GERS, the present study found that age, anxiety, the habit of lying flat at rest immediately after meals, hypertension, and albumin levels were also independently correlated with GERS. Hu et $\mathrm{al}^{40}$ discovered that there may be a link between gastroesophageal reflux disease and hypertension through observing changes in blood pressure before and after antireflux surgery in gastroesophageal reflux patients. There was a significant 
correlation between hypertension and GERD, as suggested by Li et al. ${ }^{41}$ Their findings were consistent with our study. In addition, we found a negative association between albumin levels and gastroesophageal reflux symptoms. It was possible to relate to the nutriture of patients who suffered gastroesophageal reflux symptoms. ${ }^{42}$ These findings might have certain guiding implications for the prevention and treatment of GERD.

This study had some advantages. First, it was the first study to apply the new positive diagnostic criteria for MAFLD in investigating the relationship between fatty liver and GERD. Second, all the subjects included in the present study were healthy subjects for physical examination, which minimized the influence of other diseases on the results. However, there were some limitations in the present study. First, the diagnosis of GERD in the present study was based on the GerdQ score only, without endoscopy or 24-hour $\mathrm{pH}$ monitoring. Second, all participants were Chinese, and the findings might not apply to other races. Third, the present study was a cross-sectional study and, therefore, could not show a causal relationship between MAFLD and GERD.

In conclusion, the present study clarified that MAFLD was an independent risk factor for GERS in Chinese patients. The pathophysiology concerning the correlation between fatty liver and GERD, as well as the molecular biological mechanisms, need to be further investigated.

\section{Ethics Approval and Consent to Participate}

The study was conducted in accordance with the Declaration of Helsinki (as was revised in 2013). The study was approved by Ethics Committee of the First Affiliated Hospital of Dalian Medical University (NO.PJKS-KY-2019-104). Written informed consent was obtained from all participants.

\section{Acknowledgments}

We are particularly grateful to all the people who have given us help on our article.

\section{Funding}

There is no funding to report.

\section{Disclosure}

The authors declare that they have no competing interests.

\section{References}

1. Younossi ZM, Koenig AB, Abdelatif D, Fazel Y, Henry L, Wymer M. Global epidemiology of nonalcoholic fatty liver disease-metaanalytic assessment of prevalence, incidence, and outcomes. Hepatology. 2016;64(1):73-84. doi:10.1002/hep.28431

2. Lonardo A, Nascimbeni F, Mantovani A, Targher G. Hypertension, diabetes, atherosclerosis and NASH: cause or consequence? J Hepatol. 2018;68(2):335-352. doi:10.1016/j.jhep.2017.09.021

3. Eslam M, Newsome PN, Sarin SK, et al. A new definition for metabolic dysfunction-associated fatty liver disease: an international expert consensus statement. J Hepatol. 2020;73(1):202-209. doi:10.1016/j.jhep.2020.03.039

4. Eslam M, Sarin SK, Wong VW, et al. The Asian Pacific Association for the Study of the liver clinical practice guidelines for the diagnosis and management of metabolic associated fatty liver disease. Hepatol Int. 2020;14(6):889-919. doi:10.1007/s12072-020-10094-2

5. Vakil N, van Zanten SV, Kahrilas P, Dent J, Jones R; Global Consensus Group. The Montreal definition and classification of gastroesophageal reflux disease: a global evidence-based consensus. $\mathrm{Am}$ $J$ Gastroenterol. 2006;101(8):1900-1943. doi:10.1111/j.15720241.2006.00630.x

6. El-Serag HB, Sweet S, Winchester CC, Dent J. Update on the epidemiology of gastro-oesophageal reflux disease: a systematic review. Gut. 2014;63(6):871-880. doi:10.1136/gutjnl-2012-304269

7. Ronkainen J, Agréus L. Epidemiology of reflux symptoms and GORD. Best Pract Res Clin Gastroenterol. 2013;27(3):325-337. doi:10.1016/j.bpg.2013.06.008

8. Drahos J, Ricker W, Parsons R, Pfeiffer RM, Warren JL, Cook MB. Metabolic syndrome increases risk of Barrett esophagus in the absence of gastroesophageal reflux: an analysis of SEER-medicare data. J Clin Gastroenterol. 2015;49(4):282-288. doi:10.1097/ MCG.0000000000000119

9. Niigaki M, Adachi K, Hirakawa K, Furuta K, Kinoshita Y. Association between metabolic syndrome and prevalence of gastroesophageal reflux disease in a health screening facility in Japan. $J$ Gastroenterol. 2013;48(4):463-472. doi:10.1007/s00535-0120671-3

10. Kallel L, Bibani N, Fekih M, et al. Metabolic syndrome is associated with gastroesophageal reflux disease based on a 24-hour ambulatory pH monitoring. Dis Esophagus. 2011;24(3):153-159. doi:10.1111/ j.1442-2050.2010.01118.x

11. Chung SJ, Kim D, Park MJ, et al. Metabolic syndrome and visceral obesity as risk factors for reflux oesophagitis: a cross-sectional case-control study of 7078 Koreans undergoing health check-ups. Gut. 2008;57(10):1360-1365. doi:10.1136/gut.2007.147090

12. Sookoian S, Pirola CJ. Nonalcoholic fatty liver disease and metabolic syndrome: shared genetic basis of pathogenesis. Hepatology. 2016;64 (5):1417-1420. doi:10.1002/hep.28746

13. Choi JS, Kim HM, Yang YJ, Lee S, Jeong SH, Han KJ. Fatty liver disease and the risk of erosive oesophagitis in the Korean population: a cross-sectional study. BMJ Open. 2019;9(1):e023585. doi:10.1136/ bmjopen-2018-023585

14. Min YW, Kim Y, Gwak GY, et al. Non-alcoholic fatty liver disease and the development of reflux esophagitis: a cohort study. J Gastroenterol Hepatol. 2018;33(5):1053-1058. doi:10.1111/ jgh. 14042

15. Yang HJ, Chang Y, Park SK, et al. Nonalcoholic fatty liver disease is associated with increased risk of reflux esophagitis. Dig Dis Sci. 2017;62(12):3605-3613. doi:10.1007/s10620-017-4805-6

16. Hung WC, Wu JS, Sun ZJ, Lu FH, Yang YC, Chang CJ. Gender differences in the association of non-alcoholic fatty liver disease and metabolic syndrome with erosive oesophagitis: a cross-sectional study in a Taiwanese population. BMJ Open. 2016;6(11):e013106. doi:10.1136/bmjopen-2016-013106 
17. Fujiwara M, Eguchi Y, Fukumori N, et al. The symptoms of gastroesophageal reflux disease correlate with high body mass index, the aspartate aminotransferase/alanine aminotransferase ratio and insulin resistance in Japanese patients with non-alcoholic fatty liver disease. Intern Med. 2015;54(24):3099-3104. doi:10.2169/internalmedicine.54.4297

18. Hung WC, Wu JS, Yang YC, Sun ZJ, Lu FH, Chang CJ. Nonalcoholic fatty liver disease vs. obesity on the risk of erosive oesophagitis. Eur J Clin Invest. 2014;44(12):1143-1149. doi:10.1111/ eci. 12348

19. Catanzaro R, Calabrese F, Occhipinti S, et al. Nonalcoholic fatty liver disease increases risk for gastroesophageal reflux symptoms. Dig Dis Sci. 2014;59(8):1939-1945. doi:10.1007/s10620-014-3113-7

20. Miele L, Cammarota G, Vero V, et al. Non-alcoholic fatty liver disease is associated with high prevalence of gastro-oesophageal reflux symptoms. Dig Liver Dis. 2012;44(12):1032-1036. doi:10.1016/j.dld.2012.08.005

21. Fujikawa Y, Tominaga K, Fujii H, et al. High prevalence of gastroesophageal reflux symptoms in patients with non-alcoholic fatty liver disease associated with serum levels of triglyceride and cholesterol but not simple visceral obesity. Digestion. 2012;86(3):228-237. doi: $10.1159 / 000341418$

22. Jones R, Junghard O, Dent J, et al. Development of the GerdQ, a tool for the diagnosis and management of gastro-oesophageal reflux disease in primary care. Aliment Pharmacol Ther. 2009;30 (10):1030-1038. doi:10.1111/j.1365-2036.2009.04142.x

23. Dent J, Vakil N, Jones R, et al. Accuracy of the diagnosis of GORD by questionnaire, physicians and a trial of proton pump inhibitor treatment: the Diamond Study. Gut. 2010;59(6):714-721. doi:10.1136/gut.2009.200063

24. Lacy BE, Chehade R, Crowell MD. A prospective study to compare a symptom-based reflux disease questionnaire to 48-h wireless $\mathrm{pH}$ monitoring for the identification of gastroesophageal reflux (revised 2-26-11). Am J Gastroenterol. 2011;106(9):1604-1611. doi:10.1038/ ajg.2011.180

25. Jonasson C, Wernersson B, Hoff DA, Hatlebakk JG. Validation of the GerdQ questionnaire for the diagnosis of gastro-oesophageal reflux disease. Aliment Pharmacol Ther. 2013;37(5):564-572. doi:10.1111/ apt.12204

26. Suzuki H, Matsuzaki J, Okada S, Hirata K, Fukuhara S, Hibi T. Validation of the GerdQ questionnaire for the management of gastro-oesophageal reflux disease in Japan. United Eur Gastroenterol J. 2013;1(3):175-183. doi:10.1177/2050640613485238

27. Zavala-Gonzales MA, Azamar-Jacome AA, Meixueiro-Daza A, et al. Validation and diagnostic usefulness of gastroesophageal reflux disease questionnaire in a primary care level in Mexico. $J$ Neurogastroenterol Motil. 2014;20(4):475-482. doi:10.5056/jnm14014

28. Zigmond AS, Snaith RP. The hospital anxiety and depression scale. Acta Psychiatr Scand. 1983;67(6):361-370. doi:10.1111/j.16000447.1983.tb09716.x

29. Turon H, Carey M, Boyes A, Hobden B, Dilworth S, Sanson-Fisher R. Agreement between a single-item measure of anxiety and depression and the hospital anxiety and depression scale: a cross-sectional study. PLoS One. 2019;14(1):e0210111. doi:10.1371/journal.pone.0210111

30. Wondie Y, Mehnert A, Hinz A, Frey R. The Hospital Anxiety and Depression Scale (HADS) applied to Ethiopian cancer patients. PLoS One. 2020;15(12):e0243357. doi:10.1371/journal.pone.0243357
31. Westhoff-Bleck M, Winter L, Aguirre Davila L, et al. Diagnostic evaluation of the hospital depression scale (HADS) and the Beck depression inventory II (BDI-II) in adults with congenital heart disease using a structured clinical interview: impact of depression severity. Eur J Prev Cardiol. 2020;27(4):381-390. doi:10.1177/ 2047487319865055

32. Yue T, Li Q, Wang R, et al. Comparison of Hospital Anxiety and Depression Scale (HADS) and Zung Self-Rating Anxiety/Depression Scale (SAS/SDS) in evaluating anxiety and depression in patients with psoriatic arthritis. Dermatology. 2020;236(2):170-178. doi: $10.1159 / 000498848$

33. Obesity: preventing and managing the global epidemic. Report of a WHO consultation. World Health Organ Tech Rep Ser. 2000;894:i253.

34. Sun W, Zhang D, Sun J, et al. Association between non-alcoholic fatty liver disease and autonomic dysfunction in a Chinese population. QJM. 2015;108(8):617-624. doi:10.1093/qjmed/hcv006

35. Sabath E, Báez-Ruiz A, Buijs RM. Non-alcoholic fatty liver disease as a consequence of autonomic imbalance and circadian desynchronization. Obes Rev. 2015;16(10):871-882. doi:10.1111/ obr. 12308

36. Gehrke N, Schattenberg JM. Metabolic inflammation-a role for hepatic inflammatory pathways as drivers of comorbidities in nonalcoholic fatty liver disease? Gastroenterology. 2020;158(7):1929-1947. e6.

37. Jorge ASB, Andrade JMO, Paraíso AF, et al. Body mass index and the visceral adipose tissue expression of IL-6 and TNF-alpha are associated with the morphological severity of non-alcoholic fatty liver disease in individuals with class III obesity. Obes Res Clin Pract. 2018;12(Suppl 2):1-8. doi:10.1016/j. orcp.2016.03.009

38. Nelson JE, Handa P, Aouizerat B, et al. Increased parenchymal damage and steatohepatitis in Caucasian non-alcoholic fatty liver disease patients with common IL1B and IL6 polymorphisms. Aliment Pharmacol Ther. 2016;44(11-12):1253-1264. doi:10.1111/ apt.13824

39. Rieder F, Biancani P, Harnett K, Yerian L, Falk GW. Inflammatory mediators in gastroesophageal reflux disease: impact on esophageal motility, fibrosis, and carcinogenesis. Am J Physiol Gastrointest Liver Physiol. 2010;298(5):G571-G581. doi:10.1152/ajpgi.00454.2009

40. Hu Z, Chen M, Wu J, et al. Improved control of hypertension following laparoscopic fundoplication for gastroesophageal reflux disease. Front Med. 2017;11(1):68-73. doi:10.1007/s11684-0160490-7

41. Li ZT, Ji F, Han XW, Wang L, Yue YQ, Wang ZG. The role of gastroesophageal reflux in provoking high blood pressure episodes in patients with hypertension. J Clin Gastroenterol. 2018;52 (8):685-690.

42. Oh C, Youn JK, Han JW, Kim HY, Jung SE. Analysis of growth, nutritional status and hospital visitation scores associated with reflux after nissen fundoplication in neurologically impaired children with gastroesophageal reflux. World J Surg. 2018;42(5):1463-1468. doi:10.1007/s00268-017-4276-0 


\section{Publish your work in this journal}

Diabetes, Metabolic Syndrome and Obesity: Targets and Therapy is an international, peer-reviewed open-access journal committed to the rapid publication of the latest laboratory and clinical findings in the fields of diabetes, metabolic syndrome and obesity research. Original research, review, case reports, hypothesis formation, expert opinion and commentaries are all considered for publication. The manuscript management system is completely online and includes a very quick and fair peer-review system, which is all easy to use. Visit http://www.dovepress.com/testimonials.php to read real quotes from published authors.

Submit your manuscript here: https://www.dovepress.com/diabetes-metabolic-syndrome-and-obesity-targets-and-therapy-journal 